\title{
Quantitative lung $T$ cell responses aid the rapid diagnosis of pulmonary tuberculosis
}

\author{
K Dheda, ${ }^{1,2,3}$ R N van Zyl-Smit, ${ }^{1}$ R Meldau, ${ }^{1}$ S Meldau, ${ }^{1}$ G Symons, ${ }^{4}$ H Khalfey, ${ }^{4}$ \\ N Govender, ${ }^{4}$ V Rosu, ${ }^{5}$ L A Sechi, ${ }^{5}$ A Maredza, ${ }^{1}$ P Semple, ${ }^{1}$ A Whitelaw, ${ }^{6}$ \\ H Wainwright, ${ }^{7}$ M Badri, ${ }^{4}$ R Dawson, ${ }^{1}$ E D Bateman, ${ }^{1}$ A Zumla ${ }^{3}$
}

- Additional methods, figures and tables are published online only at http://thorax.bmj.com/ content/vol64/issue10

${ }^{1}$ Lung Infection and Immunity Unit \& CTBRI, UCT Lung Institute \& Division of Pulmonology, Department of Medicine, University of Cape Town, South Africa; ${ }^{2}$ Institute of Infectious Disease and Molecular Medicine, University of Cape Town, South Africa; ${ }^{3}$ Centre for Infectious Diseases and International Health, University College Medical School, London, UK; ${ }^{4}$ Department of Medicine, University of Cape Town, South Africa; ${ }^{5}$ Dipartimento di Scienze Biomediche, University of Sardinia, Sassari, Italy; ${ }^{6}$ Division of Medical Microbiology, University of Cape Town, South Africa; ${ }^{7}$ Department of Anatomical Pathology, University of Cape Town, South Africa

Correspondence to:

K Dheda, Department of

Medicine, Groote Schuur

Hospital, Observatory, Cape

Town, South Africa, 7925:

keertan.dheda@uct.ac.za

Received 10 March 2009 Accepted 19 June 2009

Published Online First 9 July 2009

\section{ABSTRACT}

Background: The diagnosis of smear-negative pulmonary tuberculosis (TB) is problematic. There are limited data on the profile of alveolar TB antigen-specific T cells, and their utility for the rapid immunodiagnosis of pulmonary TB is unclear.

Methods: Antigen-specific interferon $\gamma$ (IFN $\gamma$ ) responses to the RD- 1 antigens ESAT-6 and CFP-10 (T-SPOT.TB and QuantiFERON-TB-Gold-In-Tube), heparin-binding haemagglutinin and purified protein derivative were evaluated, using alveolar lavage cells, in 91 consecutively recruited South African patients suspected of having TB.

Results: Of 85 evaluable patients (29\% HIV+), 24, 11, 48 and 2 had definite TB, probable TB, non-TB and an uncertain diagnosis, respectively. Between 34\% (TSPOT.TB) and 41\% (QuantiFERON-TB-Gold-In-Tube) of all test results were inconclusive. Failure of the positive control was significantly higher with the QuantiFERON-TBGold-In-Tube than with T-SPOT.TB (85\% vs $46 \%$ of inconclusive results; $p=0.001)$. Using staphylococcal enterotoxin $B$, compared with phytohaemagglutinin, substantially reduced failure of the positive control $(25 \%$ to $3 \% ; p=0.02$ ). In evaluable samples, when the definite and non-TB groups were used for outcome analysis, the percentage sensitivity, specificity, positive predictive value and negative predictive value for T-SPOT.TB ( $\geqslant 20$ spots/million alveolar mononuclear cells) and QuantiFERON-TB-Gold-In-Tube (0.35 IU/ml) were 89, 94, 89 and $94 \%(n=55)$ and 55, 86, 77 and $69 \%(n=46)$, respectively. Rapid diagnosis of TB was achieved more frequently with T-SPOT.TB than with smear microscopy (14/24 (58\%) vs. 7/24 (29\%) of definite TB cases; $p=0.02)$. Heparin-binding haemagluttinin and purified protein derivative alveolar lymphocyte IFN $\gamma$ responses had poor performance outcomes.

Conclusion: Provided evaluable results are obtained, the RD-1, but not the heparin-binding haemagglutinin or purified protein derivative, alveolar lymphocyte IFN $\gamma$ ELISPOT response is a useful rapid immunodiagnostic test for TB. However, test utility in high-burden settings may be limited by the high proportion of inconclusive results.

Sputum-scarce and smear-negative tuberculosis (TB) continues to pose a diagnostic conundrum to clinicians because the yield of rapid diagnostic tools is low and obtaining representative biological samples may be challenging. Smear microscopy has a poor sensitivity, and culture results take several weeks to obtain. Empiric treatment exposes patients to prolonged treatment with toxic drugs, and, if this fails, possible unnecessary therapy for drug-resistant TB. The only alternative rapid diagnostic tool, nucleic acid amplification testing
(NAAT), has a variable and suboptimal sensitivity ( $\sim 40 \%$ in smear-negative pulmonary $\mathrm{TB}) .{ }^{1}$

Pulmonary $\mathrm{TB}$ is characterised by a robust Th1 ( $T$ helper 1 ) response, raising the prospect of rapid immunodiagnosis using TB antigen-driven alveolar $\mathrm{T}$ cell interferon $\gamma$ (IFN $\gamma$ ) responses. ${ }^{2-7}$ Alveolar purified protein derivative (PPD)-specific $\mathrm{T}$ cell responses have been investigated as a $\mathrm{TB}$ diagnostic tool. ${ }^{8}$ However, PPD may drive pulmonary memory $\mathrm{T}$ cell responses in those with latent $\mathrm{TB}$ infection $^{6}$ and/or exposure to non-tuberculous mycobacteria, and thus may have limited specificity. More recently, standardised rapid immunodiagnostic assays (T-SPOT.TB and QuantiFERONTB Gold-In-Tube (OFT-GIT)), which evaluate IFN $\gamma$ responses to the RD- 1 antigens ESAT- 6 and CFP-10, have been shown to be highly sensitive and specific tests for the diagnosis of TB infection ${ }^{9-}$ 11 but they cannot distinguish latent from active disease when using peripheral blood mononuclear cells (PBMCs). ${ }^{12}$ However, at the site of active TB, in contrast to non-tuberculous disease, there is an $\sim 10$-fold higher frequency of TB antigen-specific $\mathrm{T}$ cells. ${ }^{13-15}$ Thus, it is reasonable to hypothesise that lung-orientated rapid immunodiagnosis of $\mathrm{TB}$ is possible using cells from affected pulmonary segments. Indeed, a preliminary study performed in Europe indicated that TB could be rapidly and accurately diagnosed with the T-SPOT.TB assay using bronchoalveolar lavage mononuclear cells (BALMCs). ${ }^{16}$ However, it remains unclear whether these outcomes can be reproduced in routine clinical practice, particularly in a high-burden $\mathrm{TB}$ and HIV setting where it would be most useful.

There are also several other unresolved questions. It is unclear whether alveolar antigenspecific responses distinguish between active and latent TB infection, how HIV infection impacts upon pulmonary immune responses and which antigens are most useful. The comparative diagnostic utility of the different standardised RD-1 assays (OFT-GIT and T-SPOT.TB) and other novel antigens (heparin-binding haemagglutinin (HBHA)) have not previously been evaluated using BALMCs. HBHA, a novel and relatively TB-specific antigen, may distinguish latent from active disease $^{17} 18$ and may therefore be particularly well suited to pulmonary immunodiagnosis. However, in general, data about the immunological profile of $\mathrm{TB}$ antigen-specific pulmonary $\mathrm{T}$ cells are limited, ${ }^{8} 1516$ and the optimal cell number and cut-off point needed for reliable immunodiagnosis are unclear. 
To address these questions we prospectively evaluated antigen-specific IFN $\gamma$ responses, including a head-to-head comparison between the T-SPOT.TB (RD-1 ELISPOT) and OFT-GIT (RD-1 ELISA) assays, and HBHA and PPD responses, using whole blood and BALMCs, in a well categorised cohort of 91 patients from Cape Town, South Africa suspected of having pulmonary $\mathrm{TB}$.

\section{METHODS}

\section{Patient recruitment and investigation}

Ninety-one consecutive patients with suspected pulmonary TB who were unable to expectorate sputum (sputum-scarce) or who were smear negative were prospectively recruited at Groote Schuur Hospital in Cape Town, South Africa (a study plan is outlined in fig 1). The study protocol was approved by the University of Cape Town Ethics Committee. Six patients were excluded (fig 1). There were thus 85 patients from whom evaluable samples were obtained. All patients had a history taken, detailed physical examination performed, routine investigations including testing for HIV infection, chest $x$ ray, and bronchoscopy performed by an experienced pulmonologist under local anaesthesia and conscious sedation. Approximately $150-300 \mathrm{ml}$ of sterile saline was used to lavage a radiologically involved lung segment. The aspirate ( 100-150 ml) was collected in a sterile glass bottle. Aliquots of bronchoalveolar lavage (BAL) fluid were sent for smear microscopy, Gram and fungal staining, cell differential count, cytology for malignant cells and Pneumocystis jirovecii, bacterial and fungal culture, and culture for Mycobacterium tuberculosis (MGIT 960). When appropriate, 3-5 transbronchial biopsies were performed.

The reference standard for TB was culture positivity for $M$ tuberculosis and/or histology in keeping with $\mathrm{TB}$ (caseous necrosis or acid-fast bacilli with or without granuloma formation). Patients were thus characterised by an investigator blinded to the IFN $\gamma$ release assay (IGRA) results as (1) definite
TB (meeting the reference standard); (2) non-TB (alternative diagnosis made and not treated for $\mathrm{TB}$ ); (3) probable $\mathrm{TB}$ (empiric anti-TB treatment with clinical response but not meeting the reference standard); and (4) uncertain diagnosis (patient lost to follow-up but not treated for $\mathrm{TB}$ and no alternative diagnosis made; thus TB could not be confirmed nor excluded).

\section{Alveolar lymphocyte antigen-specific IFN $\gamma$ responses (see online supplement for more detail)}

The following alveolar antigen-specific IFN $\gamma$ responses were evaluated.

- RD-1 ELISPOT IFN $\gamma$ responses to ESAT-6/CFP-10 peptide pools (T-SPOT.TB; Oxford Immunotec, Oxford, UK) were performed using $2 \times 10^{5}$ BALMCs per well (cell number selected by performing optimisation experiments in five patients; see online data supplement for details).

- RD-1 ELISA-measured IFN $\gamma$ responses to ESAT-6/CFP-10 and TB 7.7 antigens (QuantiFERON-TB Gold-In-Tube assay; Cellestis, Victoria, Australia) were performed using $1 \times 10^{6}$ BALMCs re-suspended in $1 \mathrm{ml}$ of serum-free medium in each tube. The cell number and volume used were derived through optimisation experiments using BALMCs from 10 patients (see online data supplement).

- PPD $(10 \mu \mathrm{g} / \mathrm{ml}$; Staten Serum Institute, Copenhagen, Denmark) IFN $\gamma$ ELISPOT responses were performed in duplicate using IFN $\gamma$-pre-coated ELISPOT plates (Mabtech, Stockholm, Sweden) seeded with $2 \times 10^{5}$ BALMCs per well.

- IFN $\gamma$ ELISPOT responses to HBHA (concentration of $100 \mu \mathrm{g} / \mathrm{ml}$ derived from optimisation experiments; see online supplement) were performed in duplicate using IFN $\gamma$-pre-coated ELISPOT plates. Methylated HBHA antigen was produced in cultures of Mycobacterium smegmatis 3.38 as previously described. ${ }^{19-21}$
Figure 1 Overview of study recruitment and the laboratory assays evaluated. $\mathrm{BAL}$, bronchoalveolar lavage; HBHA, heparin-binding haemagglutinin; IRGA, interferon $\gamma$ release assay; M.tb, Mycobacterium tuberculosis; PCP, Pneumocystis carinii pneumonia; PPD, purified protein derivative; OIF GIT, QuantiFERON-TB Gold-In-Tube; $\mathrm{TB}$, tuberculosis.

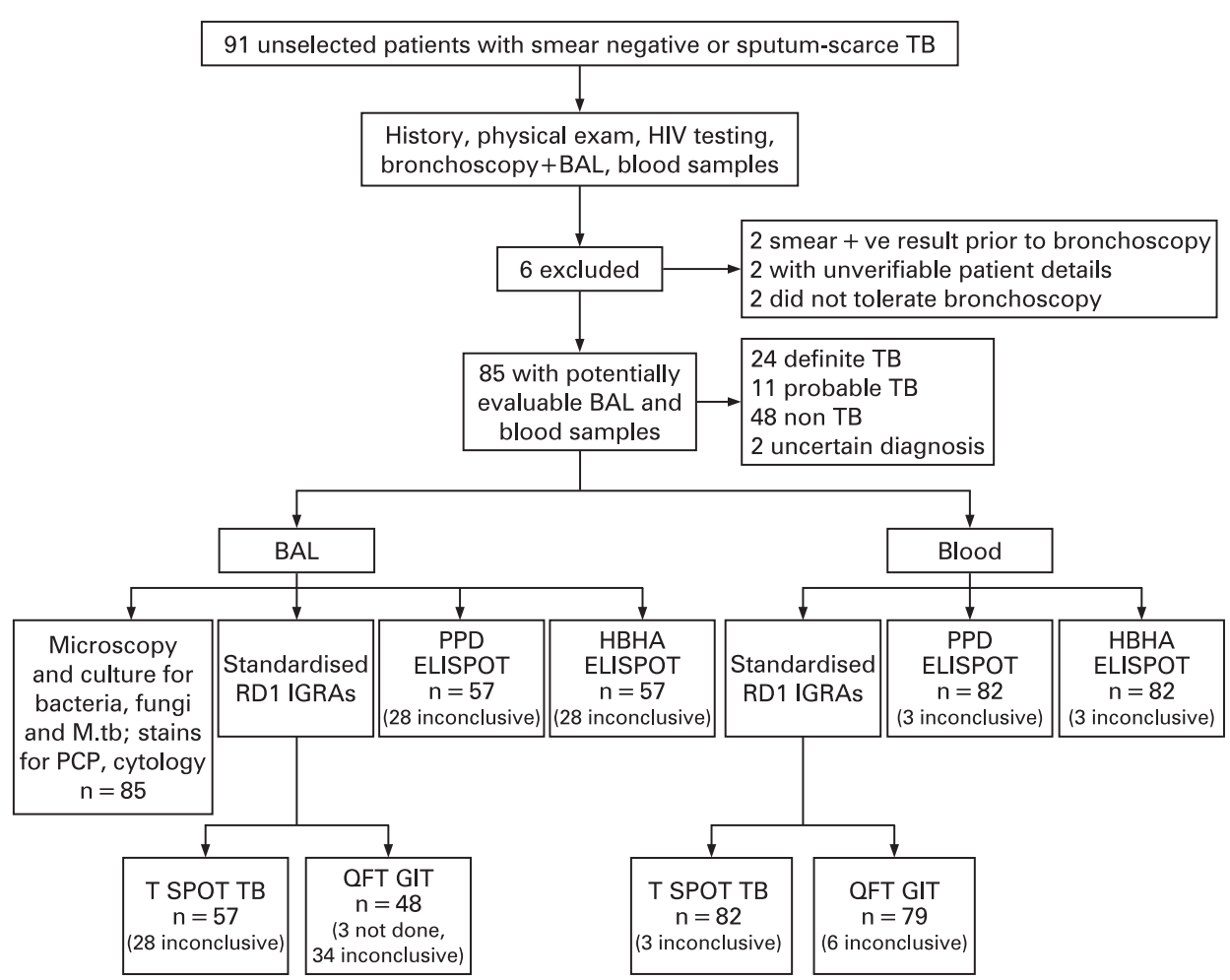


Table 1 Sociodemographic and clinical characteristics of the TB $(n=35)$ and non-TB $(n=48)$ groups

\begin{tabular}{|c|c|c|c|}
\hline & TB & No TB & \\
\hline Characteristic & n $(\%)$ & n (\%) & p Value \\
\hline Mean age (SD) & $40.71(13.6)$ & $47.38(15.7)$ & 0.05 \\
\hline Sex & & & 0.25 \\
\hline Male & $22(62.9)$ & $24(50.0)$ & \\
\hline Female & $13(37.1)$ & $24(50.0)$ & \\
\hline Ethnic group & & & 0.36 \\
\hline Black & $17(48.6)$ & $17(35.4)$ & \\
\hline Mixed race & $18(51.4)$ & $28(58.3)$ & \\
\hline White & 0 & $2(4.2)$ & \\
\hline Others & 0 & $1(2.1)$ & \\
\hline HIV status & & & 0.26 \\
\hline Positive & $13(37.1)$ & $10(20.8)$ & \\
\hline Negative & $19(54.3)$ & $33(68.8)$ & \\
\hline Unknown & $3(8.6)$ & $5(10.4)$ & \\
\hline Previous TB & & & 0.59 \\
\hline Yes & $14(40.0)$ & $18(37.5)$ & \\
\hline No & $20(57.1)$ & $26(54.2)$ & \\
\hline Unknown & $1(2.9)$ & $4(8.3)$ & \\
\hline TB contact (within 2 years) & & & 0.82 \\
\hline Yes & $6(17.1)$ & $6(12.5)$ & \\
\hline No & $24(68.6)$ & $34(70.8)$ & \\
\hline Unknown & $5(14.3)$ & $8(16.7)$ & \\
\hline Smoker & & & 0.38 \\
\hline Yes & $18(51.4)$ & $25(52.1)$ & \\
\hline No & $15(42.9)$ & $16(33.3)$ & \\
\hline Unknown & $2(5.7)$ & $7(14.6)$ & \\
\hline
\end{tabular}

Of the 85 evaluable patients two had an uncertain diagnosis. $\mathrm{TB}$, tuberculosis.

Results were deemed to be inconclusive if there were insufficient cells to perform the assay, if the positive control failed (indeterminate), if there was a high spot count/level in the negative control well/tube or, if there was high background discoloration of the ELISPOT wells precluding meaningful evaluation of the plate. In the second half of the study, in addition to phytohaemagglutinin (PHA), staphylococcal enterotoxin B (SEB) was used as a positive control.

\section{Peripheral blood-derived antigen-specific IFN $\gamma$ responses}

Peripheral blood IFN $\gamma$ responses (T-SPOT.TB, OFT-GIT, PPD and $\mathrm{HBHA}$ ) were evaluated in each patient according to the methods already described.

Details of the statistical methods used are presented in the online repository. The study satisfies the Standards for Reporting of Diagnostic Accuracy (STARD initiative) checklist. ${ }^{22}$

\section{RESULTS}

\section{Demographic and clinical details}

Demographic characteristics of the recruited patients are shown in table 1. Six patients were excluded from further analysis; thus there were 85 patients from whom evaluable cellular samples were obtained. Of the 85 patients, 24, 11, 48 and 2 had definite, probable, non-TB and an uncertain diagnosis, respectively. Of the tested patients (23/78), 29\% were HIV positive.

Smear microscopy, BAL fluid culture and transbronchial biopsies (tissue culture and histology) were positive in 7, 23 and 1 of the 24 definite TB cases, respectively, and, by definition, in none of the non-TB cases. None of the probable TB cases was culture or biopsy positive but all were treated empirically for $\mathrm{TB}$ based on clinical suspicion; all responded to anti-TB treatment.
The most common causes of pulmonary infiltrates in the nonTB group are outlined in the online data supplement. The median BAL fluid volume recovered was $100 \mathrm{ml}$ (range 40$200 \mathrm{ml})$.

\section{RD1-driven ELISA and ELISPOT assays}

Of the 85 evaluable BAL samples, the OFT-GIT assay was not performed in 3 patients, and was inconclusive (no interpretable result generated) in $34 / 82(41 \%)$ patients, thus leaving 48 samples with evaluable results. In contrast, the T-SPOT.TB was inconclusive in 28/83 (34\%) patients, thus leaving 55 samples with evaluable results. A summary of the inconclusive BAL results is given in table 2 (full details are contained in the online supplement; table E8). Inconclusive results were defined as one of the following: (1) failed positive control; (2) failed negative control (high IFN $\gamma$ level or spot count as defined by the manufacturer); (3) isolation of an inadequate number of cells (as defined in the Methods section) to perform the assay; or (4) high background discoloration of the well precluding determination of the result (applicable to the ELISPOT assay only).

In 35 ELISPOT assays, paired positive controls (PHA and SEB; positive, negative or indeterminate) results were available; of these significantly more PHA-driven than SEB-driven positive controls failed $\left(1 / 28(3 \%)\right.$ vs $7 / 28(25 \%) ; p=0.02 ; \chi^{2}$; table E2 online supplement). In the analysis presented here a failed PHA but functional SEB control was accepted as a valid result. High spot counts in the negative control well occurred in a higher proportion of HIV-positive versus negative subjects (54\% vs $22 \% p=0.01)$, but was not associated with a history of previous TB or BAL bacterial or fungal culture results.

The assay-specific (OFT-GIT and T-SPOT.TB) sensitivity, specificity, negative and positive predictive values, and area 
Table 2 Summary of the number of patients recruited, assay-specific inconclusive IGRA results, and the final number of participants used in the analysis

\begin{tabular}{|c|c|c|c|c|}
\hline \multirow[b]{2}{*}{$\begin{array}{l}\text { Diagnostic category and } \\
\text { total number recruited* }\end{array}$} & \multicolumn{2}{|c|}{ Inconclusive results } & \multicolumn{2}{|c|}{$\begin{array}{l}\text { Final number used for } \\
\text { outcome analysis }\end{array}$} \\
\hline & $\begin{array}{l}\text { SPOT.TB, } \\
\text { n (\%) }\end{array}$ & $\begin{array}{l}\text { OFT-GIT, } \\
\text { n (\%) }\end{array}$ & T-SPOT.TB & OFT-GIT \\
\hline Definite TB $(n=24)$ & $6(25)$ & $4(17)$ & 18 & $18 \dagger$ \\
\hline Probable TB $(n=11)$ & $6(45)$ & $3(17)$ & 5 & $7 \dagger$ \\
\hline $\begin{array}{l}\text { Definite and probable TB } \\
(\mathrm{n}=35)\end{array}$ & $12(34)$ & $7(23)$ & 23 & $25 \dagger$ \\
\hline Non-TB $(n=48)$ & $16(33)$ & $27(56)$ & 32 & 21 \\
\hline Total $^{*}(n=83)$ & $28(34)$ & $34(41)$ & 55 & $46 \dagger$ \\
\hline
\end{tabular}

*Two patients with an uncertain diagnosis were excluded from the table. $\uparrow$ Tests not performed (definite $\mathrm{TB}=2$; probable $\mathrm{TB}=1$; definite and probable $\mathrm{TB}=3$; total $=3$ ).

IRGA, interferon $\gamma$ release assay; OFT-GIT, QuantiFERON-TB Gold-In-Tube; $\mathrm{TB}$, tuberculosis.

under the receiver operating characteristic (ROC) of BAL samples are shown in table 3 (three cut-off points are shown: manufacturer-recommended for peripheral blood and two area under the curve (AUC)-derived cut-off points for optimal/high sensitivity and/or specificity). In evaluable samples, when the definite and non-TB groups were used for outcome analysis, the percentage sensitivity, specificity, positive predictive value and negative predictive value for T-SPOT.TB $(\geqslant 20$ spots $/$ million mononuclear cells) and OFT-GIT (0.35 IU/ml) were 89, 94, 89, $94 \%(n=55)$ and $55,86,77$ and $69 \%(n=48)$, respectively. Rapid diagnosis of TB was achieved more frequently with $\mathrm{T}$ SPOT.TB than with smear microscopy (14/24 (58\%) vs $7 /$ $24(29 \%)$ of definite TB cases; $p=0.02)$. Also shown in table 3 are comparative values when peripheral blood RD-1 responses were evaluated. Outcome values for BAL and blood RD1 responses when the definite and probable $\mathrm{TB}$ groups were combined are shown in table E3 in the online data supplement. Scatter plots and AUC of the ROC of the RD-1 ELISPOT and ELISA BALMC IFN $\gamma$ responses are shown fig $2 \mathrm{~A}$ and $\mathrm{B}$, respectively. There was moderate agreement between $\mathrm{T}$ SPOT.TB and OFT-GIT (76\% agreement, $\kappa=0.47, p=0.001$ ).

Clinical details and laboratory results of non-TB patients with a positive BAL T-SPOT.TB or QFT-GIT are shown in table $\mathrm{E} 4$, whilst clinical details and laboratory results of all definite and probable TB patients with a negative BAL T-SPOT.TB or OFT-GIT are shown in table E5 (online data supplement). In an attempt to improve the discriminatory value of the ELISPOT assay we compared the IFN $\gamma$ BALMC/PBMC ratio in the TB and non-TB groups. We hypothesised that patients with $T B$ would have a higher proportion of antigen-specific lymphocytes at the site of disease. Whilst this ratio had a high diagnostic accuracy (AUC $=0.87 ; 93 \%$ sensitivity and $71 \%$ specificity at a cut-off point of $>0.092$; fig E4, online data supplement) it was no better than the BAL ELISPOT alone. HIV status did not impact on RD-1 ELISPOT or ELISA assay outcomes (see online data supplement; fig E5).

\section{PPD and HBHA ELISPOT assay}

Sensitivities and specificity of the BAL and blood PPD- and HBHA-driven ELISPOT assay using the definite and non-TB groups, and when definite and probable TB groups were combined, and depending on the cut-off point used, are shown in tables E6 and E7 (online data supplement). Outcome values when the definite and probable $\mathrm{TB}$ groups were combined are shown in table E4 (online data supplement). Scatter plots and AUC of the ROC of the PPD- and HBHA-driven ELISPOT BALMC IFN $\gamma$ responses are shown fig $2 \mathrm{C}$ and $\mathrm{D}$, respectively.

\section{DISCUSSION}

There are limited data about the profile of alveolar TB antigenspecific (ESAT-6, CFP-10, TB 7.7 and HBHA) immune responses, ${ }^{71516}$ and the utility and feasibility of a lungorientated immunological approach for the rapid diagnosis of $\mathrm{TB}$ requires clarification. To our knowledge this is the first comparative diagnostic study of standardised antigen-specific (ESAT-6, CFP-10, TB 7.7 and HBHA) and non-specific (PPD) pulmonary lymphocyte responses. We found that although the

Table 3 Performance outcomes (95\% CI) of IRGAs (T-SPOT.TB, OFT-GIT) using alveolar lavage cells and whole blood, at different cut-off points, in patients suspected of having pulmonary TB (definite TB $(n=24)$ and non-TB groups $(n=48)$ used in the analysis).

\begin{tabular}{|c|c|c|c|c|c|c|c|}
\hline & Cut-off point & Sensitivity & Specificity & PPV & NPV & Accuracy & AUC \\
\hline \multirow{2}{*}{$\begin{array}{l}\text { T-SPOT.TB (combined RD-1 } \\
\text { antigen) }\end{array}$} & $\geqslant 30 \mathrm{SFC}^{*}$ & $78 \%(55-91)$ & $94 \%(80-98)$ & $89 \%(64-97)$ & $88 \%(74-95)$ & $88 \%(76-94)$ & $\mathrm{n} / \mathrm{a}$ \\
\hline & $\geqslant 20 \mathrm{SFC}$ & $88 \%(67-97)$ & $94 \%(80-98)$ & $89 \%(67-97)$ & $94 \%(80-98)$ & $92 \%(81-97)$ & \\
\hline T-SPOT.TB & $\geqslant 30 \mathrm{SFC}^{*}$ & $78 \%(55-91)$ & $94 \%(80-98)$ & $88 \%(64-97)$ & $88 \%(74-95)$ & $88 \%(76-94)$ & 0.96 \\
\hline ESAT-6 & $\geqslant 50 \mathrm{SFC} \dagger$ & $72 \%(49-88)$ & $97 \%(84-99)$ & $93 \%(69-99)$ & $86 \%(71-94)$ & $88 \%(76-94)$ & \\
\hline CFP-10 & $\geqslant 70 \mathrm{SFC} \uparrow$ & $61 \%(39-80)$ & $97 \%(84-99)$ & $92 \%(65-99)$ & $82 \%(67-91)$ & $84 \%(71-92)$ & \\
\hline \multirow[t]{3}{*}{ QFT-GIT } & $>0.35 \mathrm{IU} / \mathrm{ml} \mathrm{l}^{*}$ & $55 \%(34-75)$ & $86 \%(65-95)$ & $77 \%(5-92)$ & $69 \%(50-84)$ & $72 \%(56-83)$ & 0.77 \\
\hline & $>0.045 \mathrm{IU} / \mathrm{ml} \dagger$ & $83 \%(61-94)$ & $76 \%(55-89)$ & $75 \%(53-89)$ & $84 \%(62-95)$ & $79 \%(64-89)$ & \\
\hline & $>6.2 \mathrm{IU} / \mathrm{ml}$ & $28 \%(13-51)$ & $95 \%(77-99)$ & $83 \%(44-97)$ & $61 \%(44-75)$ & $64 \%(48-77)$ & \\
\hline \multicolumn{8}{|l|}{ Peripheral whole blood } \\
\hline
\end{tabular}

Forty T-SPOT.TB (SFCs per $10^{6}$ alveolar mononuclear cells) and 39 OFT-GIT (IU/ml) assays were performed.

The results reflecting outcomes when the definite and probable groups are combined are shown in the online data supplement.

* Manufacturer-recommended cut-off point for peripheral blood of $>20$ SFCs per million mononuclear cells in either the ESAT-6- or CFP-10-containing wells

$\dagger$ AUC-derived cut-off point with a high specificity at the expense of sensitivity.

:AUC-derived cut-off point with the best possible specificity at a high sensitivity.

AUC, area under the curve; IRGA, interferon $\gamma$ release assay; $n / a$, not applicable (this is a dichotomised variable and hence the AUC cannot be calculated); NPV, negative predictive value; PPV, positive predictive value; QFT-GIT, QuantiFERON-TB Gold-In-Tube; SFC, spot-forming cell; TB, tuberculosis. 

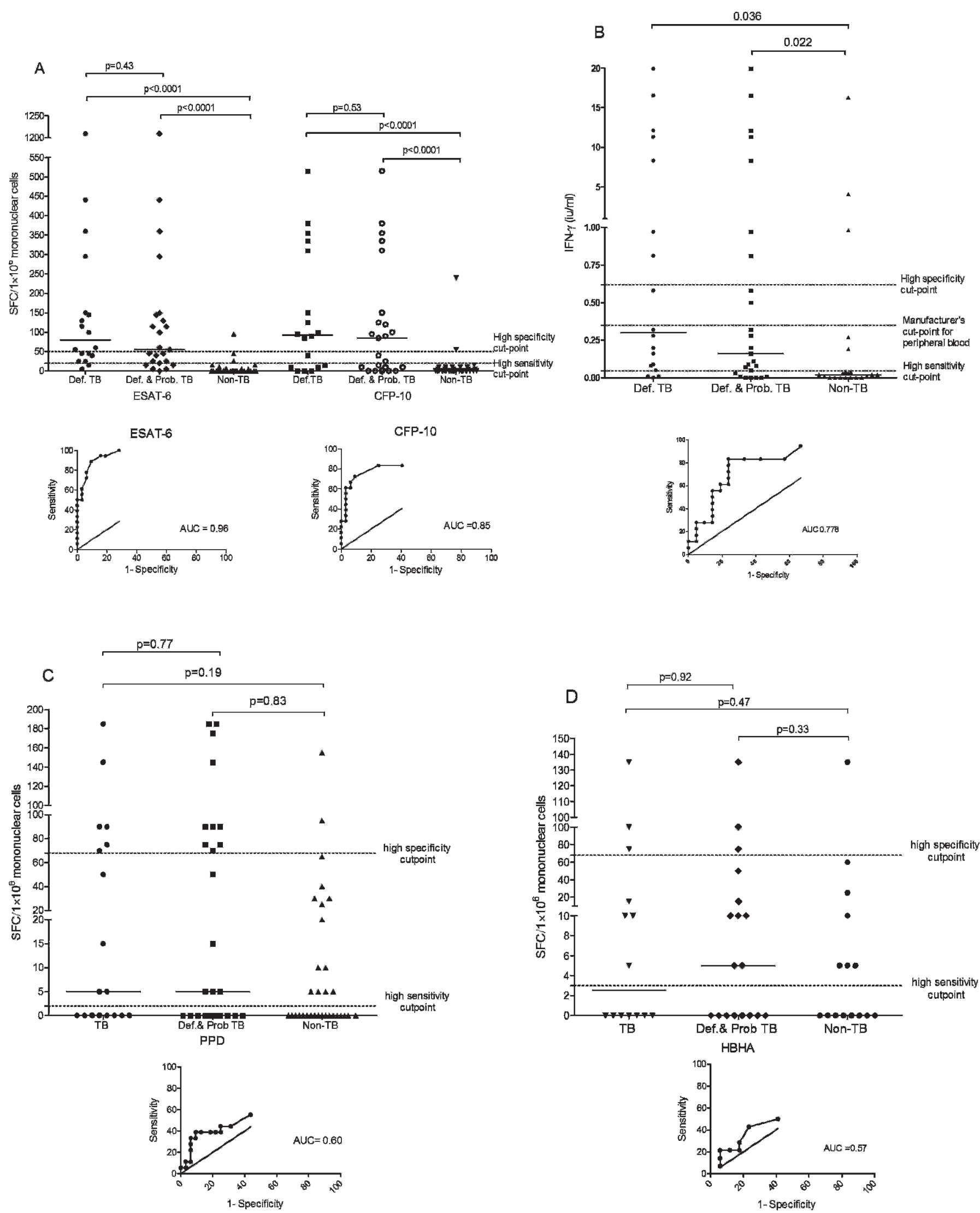

Figure 2 Scatter plots of alveolar lavage mononuclear cell RD-1-driven ELISPOT (T-SPOT.TB (A) ELISA (QuantiFERON-TB Gold-In-Tube) responses (B), purified protein derivative (PPD)-driven ELISPOT responses (C) and heparin-binding haemagglutinin (HBHA)-driven ELISPOT responses (D) in the tuberculosis (TB), combination of definite (Def.) and probable (Prob.) TB, and non-TB groups, respectively. Area under the curve (AUC)-derived cut-off points favouring a high sensitivity or specificity are shown. The AUC receiver operating characteristic (ROC) for each antigen used in the ELISPOT assay and for the ELISA (QuantiFERON-TB Gold-In-Tube) assay is depicted below each graph. SFC, spot-forming cell. 
ELISPOT assay was inconclusive in almost a third of cases, when an evaluable result was generated, it had a high sensitivity and specificity. Moreover, TB was rapidly diagnosed more frequently by the ELISPOT assay than by smear microscopy.

Although the specificity of the RD-1 ELISPOT assay was high ( $94 \%$ at a cut-off point of $\geqslant 20$ spot-forming cells (SFCs) $/ 10^{6}$ BALMCs) the sensitivity in the definite TB group was $88 \%$. The only published study examining pulmonary $\mathrm{RD}-1$ responses using the T-SPOT.TB assay found a sensitivity of $100 \%$ using a cut-off point of $\geqslant 30$ spots per million BALMCs. ${ }^{16}$ However, this preliminary study had a small sample size $(n=8$ definite and $\mathrm{n}=4$ probable TB) and was performed in a setting of low TB and HIV prevalence. Factors such as a larger sample size, HIV co-infection (though we found no difference when results were stratified by HIV status), host human leucocyte antigen (HLA) phenotype, strain differences, immunosuppressive cytokines (eg, interleukin-10 and transforming growth factor $\beta^{23}$ ), immunoregulatory $\mathrm{T}$ cells, ${ }^{24}$ bronchoscopic technique and our propensity to treat empirically for TB may have all contributed to the greater variability in sensitivity in our study. In keeping with our observations, a recent study, using flow cytometry, showed that $19 \%$ of definite TB cases had no measurable ESAT6 protein-specific lung immune responses. ${ }^{8}$ There were 5 out of 48 non-TB patients ( 2 with lung carcinoma and 2 HIV positive) in whom the BAL ELISPOT IGRAs were positive. Although the relationship between lung cancer and TB is well described, ${ }^{25}$ and we cannot be certain that concomitant TB was not present, on follow-up we found no evidence of TB. Other possible explanations include transient or latent infection.

Test outcomes were poorer for the OFT-GIT assay despite using $1 \times 10^{6}$ cells per tube. The reasons for this are unclear. Varying the cut-off point or the volume of medium in the OFTGIT tube did not meaningfully improve results. We therefore presume that performance was poorer because the ELISA is inherently a less sensitive immunological technique. We cannot exclude the possibility that the OFT-GIT-specific antigen, TB 7.7, may be driving inhibitory pathways or immunosuppressive regulatory $\mathrm{T}$ cells. ${ }^{24}$

A major practical limitation of the BAL assays was the high proportion of inconclusive results (34-41\%). The most common cause was a non-functional positive control. There was no correlation with HIV status. Immunosuppressive cytokines $^{23} 2627$ or cells, including immunosuppressive pulmonary macrophages and regulatory $\mathrm{T}$ cells, ${ }^{24}$ may explain these findings. We estimate that if SEB was used as a positive control throughout the study then the proportion of inconclusive RD-1 ELISPOT results would have dropped from $34 \%$ to $25 \%$. Further studies are now required to determine whether user-friendly depletion of immunosuppressive cells can further improve the performance outcome. This would make the assay clinically more useful. The second most common reason for inconclusive results was a high spot count in the negative control. Adjustment of the negative control ELISPOT cut-off point did not significantly improve outcomes without compromising sensitivity. Notably, $22 \%$ of the cohort had evidence of colonisation (positive bacterial or fungal culture in the absence of clinical disease), and high spot counts were significantly associated with HIV infection. Thus, although not proven, it is likely that airway pathogen colonisation, more common in high HIV and resource-poor settings, induces high spot counts in the T-SPOT.TB assay by activating the BAL alveolar T cells. Other reasons for inconclusive results were significant discoloration (blue-out) of the ELISPOT wells and low numbers of cells harvested at bronchoscopy, particularly in technically difficult procedures.

Interestingly, there were many non-TB patients with presumed latent TB infection (blood RD-1 IGRA positive) who were BAL RD-1 IGRA negative. How do we explain these findings given that the lung is a major site of latent infection? In contrast to the peripheral blood compartment, which contains circulating lymphocytes, the alveolar compartment may not reflect what is happening in the interstitial tissue (site of infection) or mediastinal lymph nodes where the majority of $\mathrm{T}$ cells reside. Compared with the RD-1 ELISPOT assay the specificity of the PPD assay was relatively poor. A likely reason is the high exposure to environmental mycobacteria in a resource-poor setting. It is therefore, similar to the findings in a recent UK-based study $\left(76 \%\right.$ specificity), ${ }^{8}$ not useful as a rulein test in clinical practice. In contrast, the sensitivity of PPD in our study was surprisingly and inexplicably low.

HBHA, whose antigenicity is dependent on methylation of the C-terminal domain and is upregulated in murine lungs infected with $M$ tuberculosis, ${ }^{20}$ is a novel and relatively TBspecific virulence factor. ${ }^{18}$ HBHA responses may be a sensitive marker of TB infection with the ability to distinguish latent from active TB. ${ }^{17}{ }^{28}$ We therefore hypothesised that HBHA responses, which have not previously been evaluated using alveolar lavage cells, would be useful for the detection of pulmonary TB. However, HBHA, in both peripheral blood and $\mathrm{BAL}$, had poor discriminatory value. The regulatory $\mathrm{T}$ cell profile, which may modulate HBHA responses, ${ }^{29}$ is likely to be different in high burden countries and may explain these findings. The differential effect of $M$ smegmatis ${ }^{19-21}$ vs $M$ bovis $B C G$-derived HBHA on IFN $\gamma$ responses, if any, is unclear.

We have taken several steps to limit study bias, including initial experiments to determine optimum cell number and antigen concentration, blinding an experienced laboratory scientist to clinical details, performing BAL in a standardised manner, recruiting consecutive patients suspected of having $T B$ over a fixed time period and performing comparative analysis using several different antigens, assays and in both blood and BAL compartments. Nevertheless, the lack of association with HIV could be due to type 2 error. Larger studies are now required in different geographical settings to confirm these observations. Although the appropriateness of the ELISPOT assay in a resourcepoor setting may be argued, it could have utility in diagnostically challenging cases in a tertiary care setting in South Africa.

In summary, when an evaluable result is obtained, the lung RD1 ELISPOT response is a useful rapid immunodiagnostic test with a reasonably high sensitivity and specificity for pulmonary $\mathrm{TB}$. However, clinical utility may be limited by the high number of inconclusive results. A lung-orientated approach to $\mathrm{TB}$ diagnosis is thus feasible, but further work is necessary, using alternative test formats, biomarkers and/or antigens, to develop an improved assay prototype that will minimize inconclusive results.

Funding: This work was supported by the South African National Research Foundation (SARChl), the South African Medical Research Council and the UCL-UCT Collaboration Initiative.

Competing interests: None.

Ethics approval: The study protocol was approved by the University of Cape Town Ethics Committee.

Provenance and peer review: Not commissioned; externally peer reviewed.

\section{REFERENCES}

1. Ling DI, Flores LL, Riley LW, et al. Commercial nucleic-acid amplification tests for diagnosis of pulmonary tuberculosis in respiratory specimens: meta-analysis and meta-regression. PLOS ONE 2008;3:e1536. 
2. Barry SM, Lipman MC, Bannister B, et al. Purified protein derivative-activated type 1 cytokine-producing CD4+ T lymphocytes in the lung: a characteristic feature of active pulmonary and nonpulmonary tuberculosis. J Infect Dis 2003;187:243-50.

3. Condos R, Rom WN, Liu YM, et al. Local immune responses correlate with presentation and outcome in tuberculosis. Am J Respir Crit Care Med 1998;157:729-35.

4. Ribeiro-Rodrigues R, Resende CT, Johnson JL, et al. Sputum cytokine levels in patients with pulmonary tuberculosis as early markers of mycobacterial clearance. Clin Diagn Lab Immunol 2002;9:818.

5. Robinson DS, Ying S, Taylor IK, et al. Evidence for a Th1-like bronchoalveolar T-cell subset and predominance of interferon-gamma gene activation in pulmonary tuberculosis. Am J Respir Crit Care Med 1994;149:989-93.

6. Silver RF, Zukowski L, Kotake S, et al. Recruitment of antigen-specific Th1-like responses to the human lung following bronchoscopic segmental challenge with purified protein derivative of mycobacterium tuberculosis. Am J Respir Cell Mol Biol 2003;29:117-23

7. Sable SB, Goyal D, Verma I, et al. Lung and blood mononuclear cell responses of tuberculosis patients to mycobacterial proteins. Eur Respir J 2007;29:337-46.

8. Breen RA, Barry SM, Smith CJ, et al. Clinical application of a rapid lung-orientated immunoassay in individuals with possible tuberculosis. Thorax 2008;63:67-71.

9. Dheda K, Udwadia ZF, Huggett JF, et al. Utility of the antigen-specific interferongamma assay for the management of tuberculosis. Curr Opin Pulm Med 2005;11:195-202.

10. Pai M, Dheda K, Cunningham J, et al. T-cell assays for the diagnosis of latent tuberculosis infection: moving the research agenda forward. Lancet Infect Dis 2007; 7:428-38.

11. Pai M, Zwerling A, Menzies D. Systematic review: T-cell-based assays for the diagnosis of latent tuberculosis infection: an update. Ann Intern Med 2008;149:177-84.

12. Mazurek GH, Weis SE, Moonan PK, et al. Prospective comparison of the tuberculin skin test and 2 whole-blood interferon-gamma release assays in persons with suspected tuberculosis. Clin Infect Dis 2007;:45:837-45

13. Jafari C, Ernst M, Strassburg A, et al. Local immunodiagnosis of pulmonary tuberculosis by enzyme-linked immunospot. Eur Respir J 2008;31:261-5.

14. Wilkinson KA, Wilkinson RJ, Pathan A, et al. Ex vivo characterization of early secretory antigenic target 6-specific t cells at sites of active disease in pleural tuberculosis. Clin Infect Dis 2005;40:184-7.

15. Nemeth J, Winkler HM, Zwick RH, et al. Recruitment of Mycobacterium tuberculosis specific CD4+ T cells to the site of infection for diagnosis of active tuberculosis. $\mathrm{J}$ Intern Med 2009;265:163-8.
16. Jafari C, Ernst M, Kalsdorf B, et al. Rapid diagnosis of smear-negative tuberculosis by bronchoalveolar lavage enzyme-linked immunospot. Am J Respir Crit Care Med 2006;174:1048-54

17. Hougardy JM, Schepers K, Place S, et al. Heparin-binding-hemagglutinin-induced IFN-gamma release as a diagnostic tool for latent tuberculosis. PLOS ONE 2007;2:e926.

18. Locht C, Hougardy JM, Rouanet C, et al. Heparin-binding hemagglutinin, from an extrapulmonary dissemination factor to a powerful diagnostic and protective antigen against tuberculosis. Tuberculosis (Edinb) 2006;86:303-9.

19. Delogu G, Bua A, Pusceddu C, et al. Expression and purification of recombinant methylated HBHA in Mycobacterium smegmatis. FEMS Microbiol Lett 2004:239:33-9

20. Delogu G, Sanguinetti M, Posteraro B, et al. The hbha gene of Mycobacterium tuberculosis is specifically upregulated in the lungs but not in the spleens of aerogenically infected mice. Infect Immun 2006:74:3006-11.

21. Zanetti S, Bua A, Delogu G, et al. Patients with pulmonary tuberculosis develop a strong humoral response against methylated heparin-binding hemagglutinin. Clin Diagn Lab Immunol 2005;12:1135-8.

22. Bossuyt PM, Reitsma JB, Bruns DE, et al. Towards complete and accurate reporting of studies of diagnostic accuracy: the STARD initiative. Standards for reporting of diagnostic accuracy. Clin Chem 2003;49:1-6.

23. Gerosa F, Nisii C, Righetti S, et al. CD4(+) T cell clones producing both interferongamma and interleukin-10 predominate in bronchoalveolar lavages of active pulmonary tuberculosis patients. Clin Immunol 1999;92:224-34.

24. Guyot-Revol V, Innes JA, Hackforth S, et al. Regulatory T cells are expanded in blood and disease sites in tuberculosis patients. Am J Respir Crit Care Med 2006;173:803-10.

25. Ortbals DW, Marr JJ. A comparative study of tuberculous and other mycobacterial infections and their associations with malignancy. Am Rev Respir Dis 1978;117:39-45.

26. Dheda K, Chang JS, Breen RA, et al. Expression of a novel cytokine, IL-4delta2, in HIV and HIV-tuberculosis co-infection. AIDS 2005;19:1601-6.

27. Dheda K, Chang J-S, Breen RA, et al. In vivo and in vitro studies of a novel cytokine interleukin-4delta2, in pulmonary tuberculosis. Am J Resp Crit Care Med 2005;172:501-8

28. Temmerman S, Pethe K, Parra M, et al. Methylation-dependent T cell immunity to Mycobacterium tuberculosis heparin-binding hemagglutinin. Nat Med 2004;10:935-41.

29. Hougardy JM, Place S, Hildebrand M, et al. Regulatory t cells depress immune responses to protective antigens in active tuberculosis. Am J Respir Crit Care Med 2007; 176:409-16.

\section{BMJ Careers online re-launches}

BMJ Careers online has re-launched to give you an even better online experience. You'll still find our online services such as jobs, courses and careers advice, but now they're even easier to navigate and quicker to find.

New features include:

- Job alerts - you tell us how often you want to hear from us with either daily or weekly alerts

- Refined keyword searching making it easier to find exactly what you want

- Contextual display - when you search for articles or courses we'll automatically display job adverts relevant to your search

- Recruiter logos linked directly to their organisation homepage - find out more about the company before you apply

- RSS feeds now even easier to set up

Visit careers.bmj.com to find out more. 